Article

\title{
Characterization of Electrosynthesized Conjugated Polymer-Carbon Nanotube Composite: Optical Nonlinearity and Electrical Property
}

\author{
Afarin Bahrami ${ }^{1,2}$, Zainal Abidin Talib ${ }^{1, *}$, Esmaeil Shahriari ${ }^{1}$, Wan Mahmood Mat Yunus ${ }^{1}$, \\ Anuar Kasim ${ }^{3}$ and Kasra Behzad ${ }^{1}$ \\ 1 Department of Physics, Universiti Putra Malaysia, UPM, Serdang, Selangor, 43400, Malaysia; \\ E-Mails: afarin.bah@gmail.com (A.B.); esmaeil.phy@gmail.com (E.S.); \\ mahmood@science.upm.edu.my (W.M.M.Y.); kasra.behzad@gmail.com (K.B.) \\ 2 Science Faculty, Islamic Azad University, Eslamshahr Branch, 3314767653, Iran \\ 3 Department of Chemistry, Universiti Putra Malaysia, UPM, Serdang, Selangor, 43400, Malaysia; \\ E-Mail: anuar@science.upm.edu.my \\ * Author to whom correspondence should be addressed; E-Mail: zainalat @science.upm.edu.my; \\ Tel.: +603-8946-6606/6688.
}

Received: 23 October 2011 / Accepted: 19 December 2011 / Published: 16 January 2012

\begin{abstract}
The effects of multi-walled carbon nanotube (MWNT) concentration on the structural, optical and electrical properties of conjugated polymer-carbon nanotube composite are discussed. Multi-walled carbon nanotube-polypyrrole nanocomposites were synthesized by electrochemical polymerization of monomers in the presence of different amounts of MWNTs using sodium dodecylbenzensulfonate (SDBS) as surfactant at room temperature and normal pressure. Field emission scanning electron microscopy (FESEM) indicates that the polymer is wrapped around the nanotubes. Measurement of the nonlinear refractive indices $\left(\mathrm{n}_{2}\right)$ and the nonlinear absorption $(\beta)$ of the samples with different MWNT concentrations measurements were performed by a single Z-scan method using continuous wave $(\mathrm{CW})$ laser beam excitation wavelength of $\lambda=532 \mathrm{~nm}$. The results show that both nonlinear optical parameters increased with increasing the concentration of MWNTs. The third order nonlinear susceptibilities were also calculated and found to follow the same trend as $n_{2}$ and $\beta$. In addition, the conductivity of the composite film was found to increase rapidly with the increase in the MWNT concentration.
\end{abstract}


Keywords: conducting polymers; carbon nanotubes; optical properties; electrical characterization

\section{Introduction}

Conducting polymers are suitable alternative to replace metals in industry as they have the ability to withstand high electric fields with good environment stability and low cost of fabrication. Its physical properties are also not satisfactory for practical applications. This led to intensive research to achieve both high electrical conductivity and desirable optical and mechanical properties.

Polypyrrole (PPy) is a well-known conducting polymer, that has been studied by many researchers $[1,2]$. It is most frequently used in various device applications, such as in sensors $[3,4]$ microelectronic devices [5] and composite materials [6,7]. Following the first report of the synthesizing of carbon nanotubes polymer nanocomposite, [8] many attempts have been made for combination of carbon nanotubes and polymers to produce materials with superior properties [9,10]. Carbon nanotubes (CNTs) have unique structural, electrical and optical properties. They are empty cylinders with diameters less than $100 \mathrm{~nm}$ and length on the micrometer scale and one of their features is their high surface to volume ratio. There are two different main methods for polymerization that is chemical and electrochemical method. However, this work focuses on the combination of the properties of multi-walled carbon nanotube (MWNT) and polypyrrole following an electrochemical method to the formation of the nanocomposite.

Both conducting polymers and CNTs have conjugated $\pi$ bonds structure. The delocalized $\pi$ electrons in CNT and PPy can bond together in nanocomposite to reduce energy of the system to form the PPy/CNT nanocomposite [11-13] and conducting polymer MWNT nanocomposites with core-shell structure are obtained [14]. MWNTs act as a dopant in the nanocomposite deposited on the working electrode. We use MWNTs as a conductive nanomaterial inside the PPy conducting polymer for developing the physical properties due to interfacial interactions between MWNT and the conducting polymer [15-17].

In the present work, we have investigated the structural, optical and electrical properties of the conducting polymer, polypyrrole and MWNT composite films prepared using electrodeposition.

Optical characterization of the nanocomposite samples was performed using a Z-scan method. Z-scan is a well-established method for the determination of nonlinear refraction and absorption and has been widely used in material characterization because it provides not only the magnitudes of real and imaginary parts of nonlinear susceptibility, but also the sign of the real part [18,19].

A large number of papers have been published on the synthesis and characterization of CNT and PPy nanocomposite films [20,21] but to our knowledge there are a few reports on the nonlinear optical properties of the PPy/CNT nanocomposite. 


\section{Experimental Details}

In this study, the pyrrole monomer (Fluka) was distilled prior to use. Multiwalled carbon nanotubes (Nanostructure \& Amorphous Materials) and sodium dodecylbenzensulfonate (Aldrich) were of analytical grade and used without further purification.

For the preparation for electrochemical polymerization, the ITO glasses were completely washed using sonicator bath. After dissolving the sodium dodecylbenzensulfonate (SDBS) in distilled water, the MWNTs with different weight ratio were dispersed in SDBS solution and sonicated for 4 hours. The ratio of nanotubes to surfactant was 1:10. Then pyrrole was dissolved in this MWNT/SDBS solution and again ultrasonicated for another $10 \mathrm{~min}$, the monomer concentration was $0.1 \mathrm{M}$ Subsequently, the PPy/MWNT premixed solution was electropolymerized at $+0.7 \mathrm{~V}$ for $5 \mathrm{~min}$, in a three electrode electrochemical cell in which the ITO was used as a working electrode while a graphite rod and a saturated calomel electrode were used as the counter and reference electrode, respectively. The electrochemical polymerization was performed using a potentiostat (PS 605, USA) at room temperature. The current density was $0.5 \mathrm{~mA} / \mathrm{cm}^{2}$. No other salts and solvents were added to the solution. The deposited polymerized PPy/MWNT thin films were washed with water and methanol to remove the electrolyte solution and dried under vacuum at room temperature for 24 hours.

The thickness of the samples was measured using a high surface profilermeter (AMBIOS TECHNOLOGY XP-200) which has an accuracy of $\pm 10 \mathrm{~nm}$. The linear refractive index and linear transmission coefficient were measured using an ellipsometer and fiber optics spectrophotometer (OCEAN OPTICS USB4000-FL), respectively. The electrical conductivity of the nanocomposite films samples was measured by a four point probe instrument. For nonlinear properties measurements, a single beam Z-scan method with closed and open aperture arrangements was used to measure the nonlinear refractive and nonlinear absorption coefficients. The measurements were carried out at room temperature using a $\mathrm{CW}$ beam diode laser operated at $532 \mathrm{~nm}$ wavelength (Coherent Compass SDL-532-150T). The beam was focused to a small spot using a lens and the sample was moved along the $\mathrm{z}$-axis by a motorized translational stage. The power output of the laser beam measured at the focused point was $35 \mathrm{~mW}$. The transmitted light in the far field passed through the aperture and the beam intensity was recorded by a photodiode detector. The laser beam waist $\omega_{0}$ at the focus length was $24 \mu \mathrm{m}$ and the Rayleigh length was found to satisfy the basic criteria of a Z-scan experiment.

\section{Results and Discussion}

\subsection{Morphology}

Figures 1a to 1c show FESEM (FEI Nova NanoSEM 230) images of the purified MWNTs, the PPy formed by electrochemical polymerization without MWNT, and the PPy/MWNT nanocomposite formed by electropolymerization. These figures show that the diameter of CNTs has been increased by electrochemical polymerization of pyrrole monomer onto the CNT as expected, and confirms the presence of CNTs inside the films. In addition, it is obvious that coated CNTs inside the film did not have any particular direction, and they are connected to each other by means of polymer. 
The thickness of polypyrrole coated over the MWNT was in the range of 150-350 nm, and it was possible to control the thickness of the film by changing the experiment parameters, e.g., the time of polymerization or concentration of materials. From Figure 1 it is obvious the PPy formed under similar condition shows typical cauliflower morphology, with no nanocable-like morphology related to PPy/MWNT composite.

Figure 1. Field emission scanning electron microscopy (FESEM) image of (a) the Polypyrrole (PPy) formed by electrochemical polymerization without multi-walled carbon nanotube (MWNT); (b) the purified MWNTs; (c) the PPy/MWNT nanocomposite formed by electrochemical polymerization.
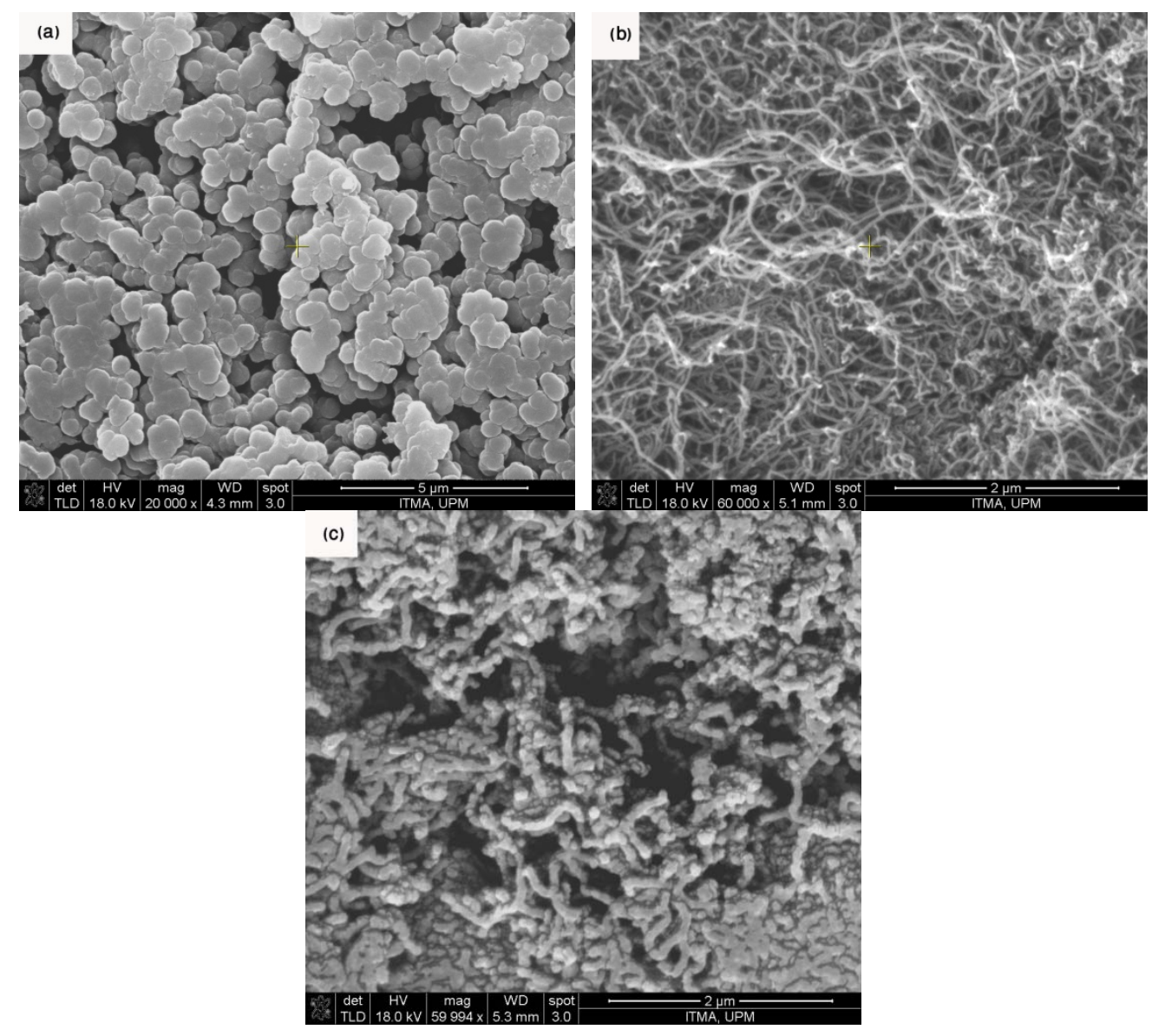

Figure 2 shows a schematic diagram of the polymerization procedure of pyrrole onto the carbon nanotubes in the presence of the surfactant. In accordance with an earlier study [22], SDBS molecules as a surfactant were absorbed firmly onto the MWNT surface, because of van der Waals intermolecular forces and has functionalized the MWNT to improve the dispersion of nanotubes inside the solution [22]. The Polypyrrole molecule could enter into this MWNT/SDBS solution and situate at the surface between surfactant and carbon nanotube [23-25]. 
Figure 2. Schematic representation of the formation of the PPy/MWNT nanocomposite in the presence of sodium dodecylbenzensulfonate (SDBS) surfactant. (a) MWNT; (b) MWNT surrounded by surfactant after sonication; (c) pyrrole adsorption on the MWNT surface; (d) polymerization of pyrrole on MWNT; (e) PPy coated MWNT after washing.

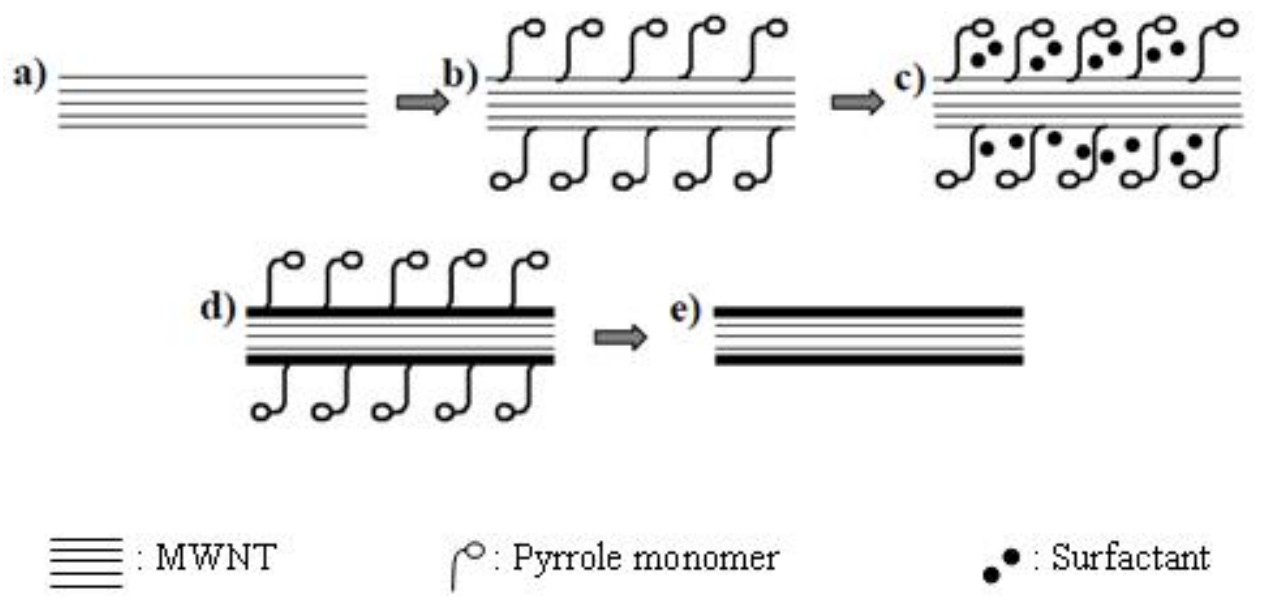

\subsection{Optical Measurements}

The absorption spectra of the samples obtained from a UV-Vis spectrophotometer (Shimadzu-UV1650PC) are shown in Figure 3. The measurements of absorption spectra were carried out at room temperature for visible wavelength ranging from $350 \mathrm{~nm}$ to $850 \mathrm{~nm}$. The linear absorption spectra values of the present samples were obtained to calculate the optical nonlinearities.

Figure 3. Absorbance spectrum of the PPy/MWNT for different MWNT (wt \%) concentrations S1: 3 wt \%; S2: 6 wt \%; S3: 9 wt \%; S4: 12 wt \%.

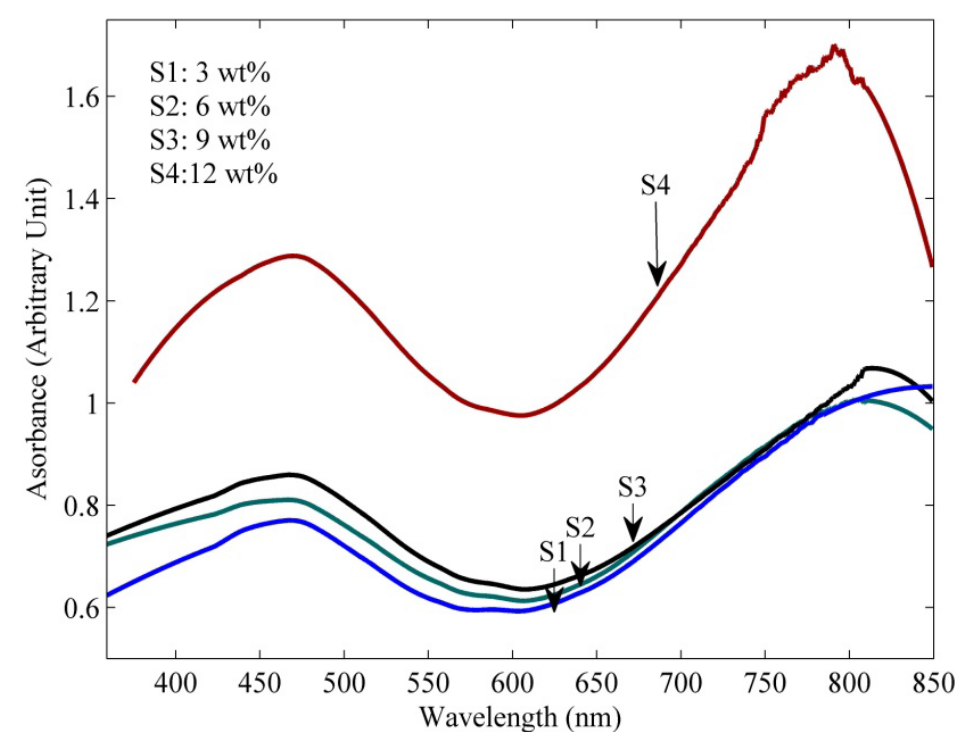

For optical nonlinearity measurements, a closed-aperture and an open-aperture have been used to estimate the nonlinear refraction coefficient and nonlinear absorption coefficient, respectively. The closed-aperture Z-scan curves for PPy/MWNT nanocomposite films are shown in Figure 4. The symbols represent the experimental data and the solid lines are theoretical fits to the closed-aperture and 
open-aperture Z-scan equations. The theoretical fits to the closed aperture (not shown) is used the standard equation given as [26].

$$
T(z, \Delta \phi)=1-\frac{4 \Delta \phi_{o} x}{\left(x^{2}+1\right)\left(x^{2}+9\right)}
$$

The nonlinear refractive index of the nanocomposite films was calculated using a simple relationship proposed by Sheik Bahaei et al. [26]:

$$
n_{2}=\Delta \phi_{0} \lambda / 2 \pi I_{0} L_{e f f}
$$

where $\lambda$ is the wavelength of the laser light, and $\mathrm{I}_{0}$ is the peak intensity within the sample. The terms $\Delta \phi_{0}$ and $L_{e f f}$ are the nonlinear phase shift and the effective thickness, respectively, given by the following relationships [26,27]:

$$
\begin{gathered}
\Delta \phi_{0}=\frac{\Delta T_{p-v}}{0.406(1-S)^{0.25}} \\
L_{\text {eff }}=\left[1-\exp \left(-\alpha_{0} L\right)\right] / \alpha_{0}
\end{gathered}
$$

Here, $L, S$, and $\alpha_{0}$ are sample thickness, aperture linear transmittance and linear absorption coefficient at wavelength $\lambda$, respectively. However, if the sample has a nonlinear refractive index and nonlinear absorption properties, the normalized transmission curve of closed-aperture data does not show a perfectly symmetrical curve. This phenomenon can be clearly seen in Figure 4.

Figure 4. Normalized Z-scan transmittance curves of closed-aperture for PPy/MWNT for different MWNT (wt \%) concentrations.

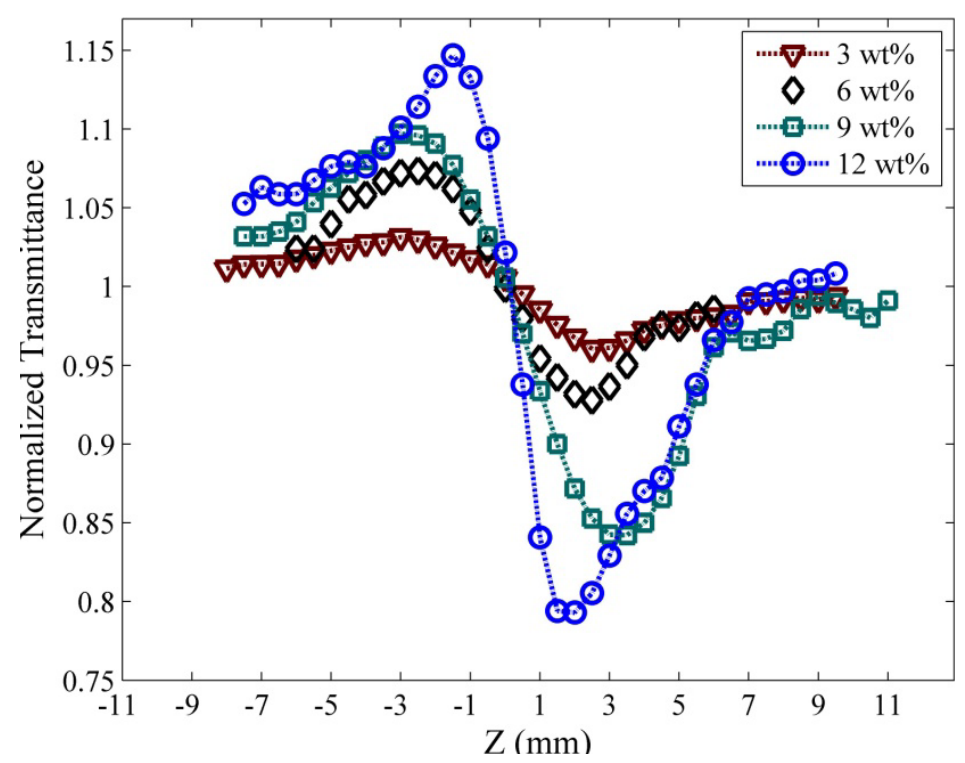

Figure 5 shows the open aperture experimental data of samples obtained for carbon nanotubes at different concentrations using $532 \mathrm{~nm}$ excitation laser beam. The nonlinear absorption coefficient $\beta$ $(\mathrm{cm} / \mathrm{W})$ can be determined from a best-fitting procedure performed on the experimental data of the open-aperture measurement using a well-known equation used by Sheik Bahae et al. [26,27]: 


$$
T(z, s=1)=\sum_{m=0}^{\infty} \frac{\left[-\beta I_{0} L_{e f f} /\left(1+z / z_{0}\right)\right]^{m}}{(m+1)^{3 / 2}}
$$

where $T(z, s=1)$ is the normalized transmittance for the open aperture $(\mathrm{OA})$ with $\mathrm{z}_{0}$ being the Rayleigh range. In this work, we used the MATLAB software for fitting the experimental data with theoretical equations 1 and 5 for closed and open aperture measurements, respectively. The nonlinear refractive index $\mathrm{n}_{2}$ and nonlinear absorption coefficient $\beta$ were used to calculate the real and imaginary parts of the third-order nonlinear optical susceptibility $\chi^{3} \quad[28,29]$ according to the following relationships:

$$
\begin{aligned}
& \operatorname{Re} \chi^{3}(e s u)=10^{-4} \frac{\varepsilon_{0} \mathrm{c}^{2} \mathrm{n}_{0}^{2}}{\pi} n_{2}\left(\mathrm{~cm}^{2} W^{-1}\right) \\
& \operatorname{Im} \chi^{3}(e s u)=10^{-2} \frac{\varepsilon_{0} \mathrm{c}^{2} \mathrm{n}_{0}^{2} \lambda}{4 \pi^{2}} \beta\left(\mathrm{cm}^{-1}\right)
\end{aligned}
$$

where $\varepsilon_{0}$ is the vacuum permittivity, and $\mathrm{c}$ is the velocity of light in a vacuum. The values of linear refractive index $\mathrm{n}_{0}$ were measured by using an ellipsometer (DRE-Dr.Riss Ellipsometerbau $\mathrm{GmbH}$ ). Thus, the absolute value of the third-order nonlinear optical susceptibility was calculated as:

$$
\left|\chi^{3}\right|=\left[\left(\operatorname{Re}\left(\chi^{3}\right)\right)^{2}+\left(\operatorname{Im}\left(\chi^{3}\right)\right)^{2}\right]^{1 / 2}
$$

The values of the nonlinear refraction coefficient $\mathrm{n}_{2}\left(\mathrm{~cm}^{2} / \mathrm{W}\right)$, nonlinear absorption coefficient $\beta(\mathrm{cm} / \mathrm{W})$ and the third-order nonlinear susceptibility obtained for the present samples are listed in Table 1.

Figure 5. Normalized Z-scan transmittance curves of open-aperture for PPy/MWNT at different MWNT (wt \%) concentrations.

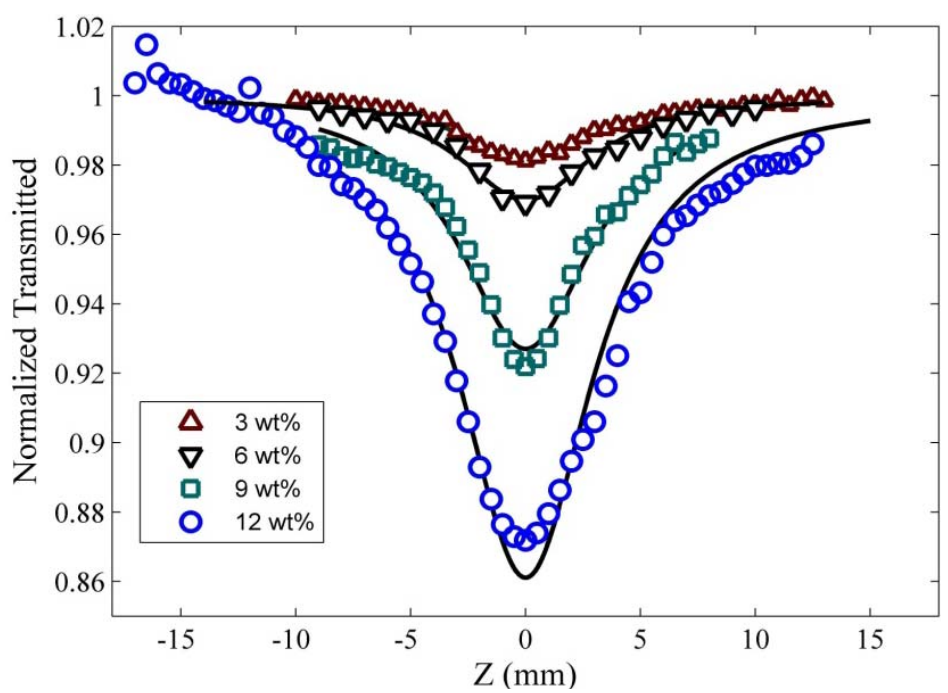


Table 1. The nonlinear optical parameters measured for PPy/MWNT at different concentrations.

\begin{tabular}{|c|c|c|c|c|c|c|}
\hline $\begin{array}{l}\text { Thin film } \\
\text { samples }\end{array}$ & $\begin{array}{c}\text { Concentration } \\
(w \mathrm{w} \%)\end{array}$ & $\begin{array}{c}\mathrm{n}_{2}\left(\mathrm{~cm}^{2} / \mathrm{W}\right) \\
\times 10^{-4}\end{array}$ & $\begin{array}{l}\beta(2 P A) \\
(\mathrm{cm} / \mathrm{W})\end{array}$ & $\begin{array}{c}\operatorname{Re}\left(\chi^{(3)}\right) \\
\times 10^{-4} \\
\end{array}$ & $\begin{array}{c}\operatorname{Im}\left(\chi^{(3)}\right) \\
\times 10^{-4} \\
\end{array}$ & $\begin{array}{l}\left|\chi^{(3)}\right| \\
\times 10^{-4} \\
\end{array}$ \\
\hline S1 & 3 & -1.721 & 0.278 & -85.461 & 0.5852 & 85.485 \\
\hline S2 & 6 & -2.872 & 0.553 & -142.612 & 1.1640 & 142.614 \\
\hline S3 & 9 & -8.211 & 1.317 & -407.951 & 2.769 & 407.959 \\
\hline S4 & 12 & -8.872 & 2.179 & -440.750 & 4.582 & 440.776 \\
\hline
\end{tabular}

The effects of concentration on nonlinear refraction and nonlinear absorption are shown in Figure 6. The nonlinear refractive index and the nonlinear absorption coefficient increased as the concentration of carbon nanotubes increased. With higher concentration of CNT more particles will interact with the laser beam and subsequently, more particles are thermally agitated resulting in enhanced effect on optical nonlinearities.

Figure 6. Variation of the nonlinear refraction coefficient and nonlinear absorption coefficient for different MWNT (wt \%) concentrations.

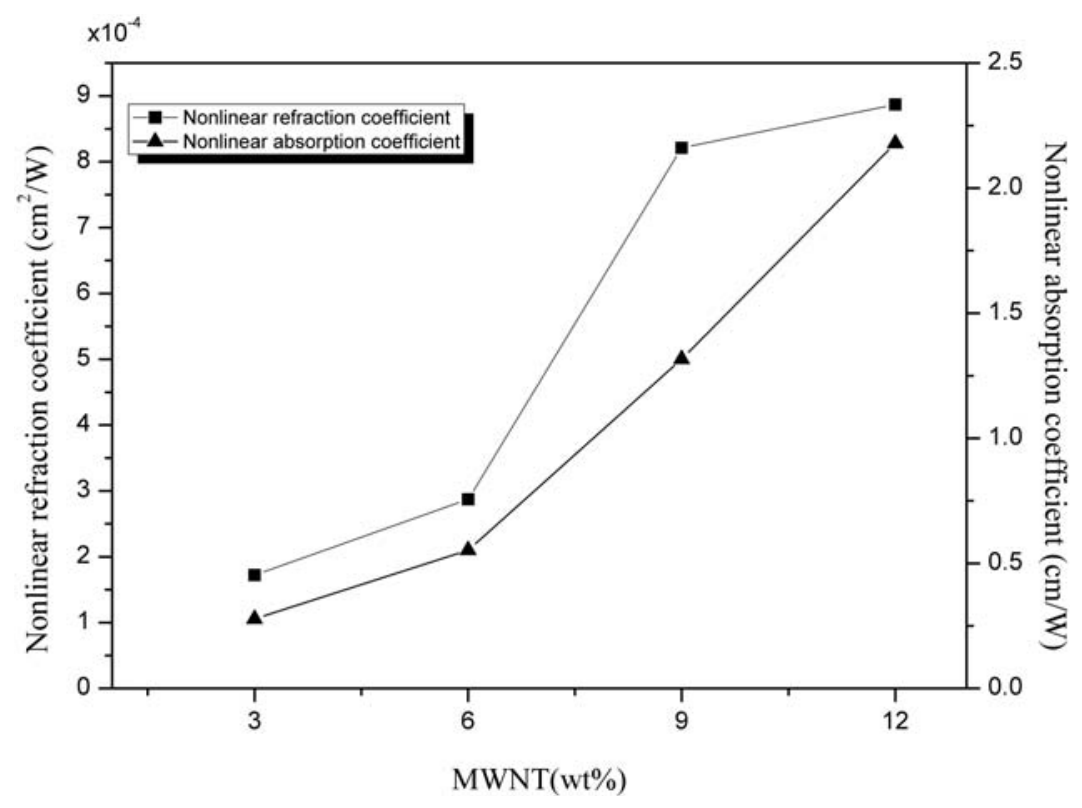

\subsection{Conductivity Studies}

The variation of the conductivity of the thin film composite versus MWNT concentration is plotted in Figure 7. The results show that conductivity increases with increasing concentration of the MWNTs in the composite. This means the nanotubes improves the conductivity of the composite film by introducing a conductive network into the polymer matrix which improves the conductivity. This may be due to the larger surface area of MWNT that serve as a conducting bridge, connecting PPy conducting domains and increasing the effective percolation [30]. One of the interesting advantages obtained, is the possibility of modulating the charge transport properties of the composite film. 
Figure 7. Variation of conductivity of the PPy/MWNT composite films for different MWNT concentration. The curve represents the best fit of the data to equation 1.

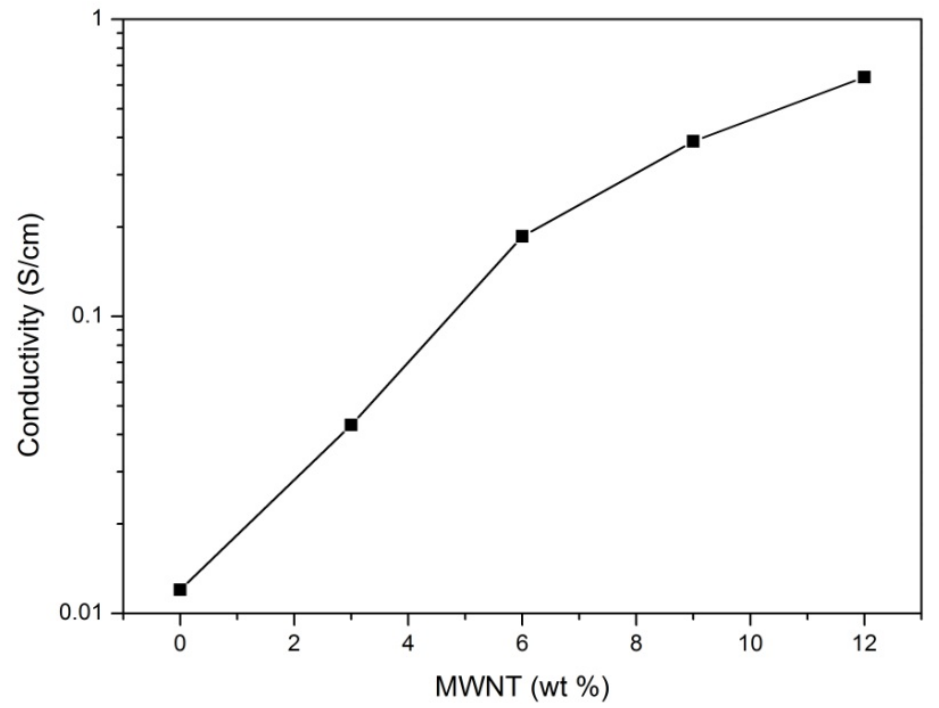

\section{Conclusions}

The presence of nanotube did not result in any significant degradation of absorption coefficient in the visible region. The nonlinear refractive index, $\mathrm{n}_{2}$, and nonlinear absorption coefficient, $\beta$, of PPy/MWNT were measured successfully for four different concentrations. The variation of the nonlinear coefficients of samples as concentrations increases was noted. In addition, third-order nonlinear optical susceptibilities were calculated using the measured values of $\mathrm{n}_{2}$ and $\beta$, and the results suggested a significant, third-order nonlinear response. The sign of the nonlinear refractive index was found to be negative and the magnitude was in the order of $10^{-4} \mathrm{~cm}^{2} / \mathrm{W}$. It can be concluded that doping of PPy with MWNT increases the conductivity of the nanocomposite which is mainly due to the introduction of conducting paths in the polymer matrix.

\section{References}

1. MacDiarmid, A.G. "Synthetic metals": A novel role for organic polymers (Nobel lecture). Angew. Chem. Int. Ed. 2001, 40, 2581-2590.

2. Skotheim, T.A. Handbook of Conducting Polymers; Marcel Dekker: New York, NY, USA, 1986; Volume 1.

3. Alizadeh, N.; Khodaei-Tazekendi, H. Linear alkylbenzenesulfonate (LAS) ion-selective electrode based on electrochemically prepared polypyrrole and PVC. Sens. Actuator B. Chem. 2001, 75, $5-10$.

4. Lin, C.W.; Hwang B.J.; Lee C.R. Methanol sensors based on the conductive polymer composites from polypyrrole and poly (vinyl alcohol). Mater. Chem. Phys. 1998, 55, 139-144.

5. Ding, H.; Ram, M.K. Applications of ordered ultrathin conducting polymeric films of polypyrrole. Supramol. Eng. Cond. Mater. 2005, 199-231.

6. Tieke, B.; Gabriel, W. Conducting polypyrrole-polyimide composite films. Polymer 1990, 31, 20-23. 
7. Pruneanu, S.; Resel, R.; Leising, G.; Brie, M.; Graupner, W.; Oniciu, L. Structural investigations on polypyrrole and poly(vinyl chloride)-polypyrrole composite films. Mater. Chem. Phys. 1997, 48, 240-245.

8. Ajayan, P.M.; Stephan, O.; Colliex, C.; Trauth, D. Aligned carbon nanotube arrays formed by cutting a polymer resin-nanotube composite. Science 1994, 265, 1212-1214.

9. Baughman, R.H.; Zakhidov, A.A.; de Heer, W.A. Carbon nanotubes-The route toward applications. Science 2002, 297, 787-792.

10. Dai, L.; Mau, A.W.H. Controlled synthesis and modification of carbon nanotubes and $\mathrm{C}_{60}$ : Carbon nanostructures for advanced polymeric composite materials. Adv. Mater. 2001, 13, 899-913.

11. Rezaul Karim, M.; Lee, C.J.; Sarwaruddin Chowdhury, A.M.; Nahar, N; Lee, M.S. Radiolytic synthesis of conducting polypyrrole/carbon nanotube composites. Mater. Lett. 2007, 61, 1688-1692.

12. Joo, J.; Kim, B.H.; Park, D.H.; Kim, H.S.; Seo, DS; Shim, J.H.; Lee, S.J.; Ryu, K.S.; Kim, K.; Jin, J.I.; Lee, T.J.; Lee, C.J. Fabrication and applications of conducting polymer nanotube, nanowire, nanohole, and double wall nanotube. Synth. Metals 2005, 153, 313-316.

13. Wang, C.; Wang, Z.; Li, M.; Li, H. Well-aligned polyaniline nano-fibril array membrane and its field emission property. Chem. Phys. Lett. 2001, 341, 431-434.

14. Yu, Y.; Ouyang, C.; Gao, Y.; Si, Z.; Chen, W.; Wang, Z.; Xue, G. Synthesis and characterization of carbon nanotube/polypyrrole core-shell nanocomposites via in situ inverse microemulsion. $J$. Polym. Sci. Part A-Polym. Chem. 2005, 43, 6105-6115.

15. Alexandrou, I.; Kymakis, E.; Amaratunga, G.A.J. Polymer-nanotube composites: Burying nanotubes improves their field emission properties. Appl. Phys. Lett. 2002, 80, 1435-1437.

16. Shi, D.; Lian, J.; He, P.; Wang, L.M.; Xiao, F.; Yang, L.; Schulz, M.J.; Mast, D.B. Plasma coating of carbon nanofibers for enhanced dispersion and interfacial bonding in polymer composites. Appl. Phys. Lett. 2003, 83, 5301-5303.

17. Lau, K.T. Interfacial bonding characteristics of nanotube/polymer composites. Chem. Phys. Lett. 2003, 370, 399-405.

18. Yang, G.; Guan, D.; Wang, W.; Wu, W.; Chen, Z. The inherent optical nonlinearities of thin silver films. Opt. Mater. 2004, 25, 439-443.

19. He, T.; Cai, Z.; Li, P.; Cheng, Y.; Mo, Y. Third-order nonlinear response of Ag/methyl orange composite thin films. J. Mod. Opt. 2008, 55, 975-983.

20. Wang, J.; Xu, Y.; Chen, X.; Sun, X. Capacitance properties of single wall carbon nanotube/polypyrrole composite films. Compos. Sci. Technol. 2007, 67, 2981-2985.

21. Brânzoi, V.; Pilan, L.; Brânzoi, F. Nanocomposite films obtained by electrochemical codeposition of conducting polymers and carbon nanotubes. Electroanalysis 2009, 21, 557-562.

22. Islam, M.F.; Rojas, E.; Bergey, D.M.; Johnson, A.T.; Yodh, A.G. High weight fraction surfactant solubilization of single-wall carbon nanotubes in water. Nano Lett. 2003, 3, 269-273.

23. Zhang, M.; Yan, Y.; Gong, K.; Mao, L.; Guo, Z.; Chen, Y. Electrostatic layer-by-layer assembled carbon nanotube multilayer film and its electrocatalytic activity for $\mathrm{O}_{2}$ reduction. Langmuir 2004, $20,8781-8785$.

24. Zhang, X.; Zhang, J.; Wang, R.; Liu, Z. Cationic surfactant directed polyaniline/CNT nanocables: Synthesis, characterization, and enhanced electrical properties. Carbon 2004, 42, 1455-1461. 
25. Zhang, X.; Zhang, J.; Wang, R.; Zhu. T.; Liu, Z. Surfactant-directed polypyrrole/cnt nanocables: Synthesis, characterization, and enhanced electrical properties. Chem. Phys. Chem. 2004, 5, 998-1002.

26. Sheik-Bahae, M.; Said, A.A.; Van Stryland, E.W. High-sensitivity, single-beam $n_{2}$ measurements. Opt. Lett. 1989, 14, 95-957.

27. Sheik-Bahae, M.; Said, A.A.; Wei, T.H.; Hagan, D.J.; Van Stryland, E.W. Sensitive measurement of optical nonlinearities using a single beam. IEEE J. Quantum Electron. 1990, 26, 760-769.

28. Yang, G.; Wang, H.; Tan, G.; Jiang, A.; Zhou, Y.; Chen, Z. Rh:BaTiO 3 thin films with large nonlinear optical properties. Appl. Opt. 2002, 41, 1729-1732.

29. Cassano, T.; Tommasi, R.; Ferrara, M.; Babudri, F.; Farinola, G.M.; Naso, F. Substituent-dependence of the optical nonlinearities in poly(2,5-dialkoxy-p-phenylenevinylene) polymers investigated by the Z-scan technique. Chem. Phys. 2001, 272, 111-118.

30. Zengin, H.; Zhou, W.; Jin, J.; Czerw, R.; Smith, D.W.; Echegoyen, L.; Carroll, D.L.; Foulger, S.H.; Ballato, J. Carbon nanotube doped polyaniline. Adv. Mater. 2002, 14, 1480-1483.

(C) 2012 by the authors; licensee MDPI, Basel, Switzerland. This article is an open access article distributed under the terms and conditions of the Creative Commons Attribution license (http://creativecommons.org/licenses/by/3.0/). 